\title{
Tc-99m (methylene diphosphonate) SPECT quantitative imaging: Impact of attenuation map generation from SPECT-non-attenuation corrected and MR images on the diagnosis of bone metastasis
}

\author{
S. Mostafapour'1, H. Arabi², F. Gholamiankhah'1, S.K. Razavi-Ratki3, \\ A.A. Parach ${ }^{*}$ \\ ${ }^{1}$ Department of Medical Physics, Faculty of medicine, Shahid Sadoughi University of Medical Sciences, Yazd, Iran \\ ${ }^{2}$ Division of Nuclear Medicine and Molecular Imaging, Department of Medical Imaging, Geneva University \\ Hospital, CH-1211 Geneva 4, Switzerland \\ ${ }^{3}$ Department of Radiology, Faculty of Medicine, Shahid Sadoughi University of Medical Sciences, Yazd, Iran
}

\section{- Original article}

\section{*Corresponding authors:}

Ali Asghar Parach, PhD.,

E-mail: aliparach@ssu.ac.ir

Revised: May 2020

Accepted: June 2020

Int. J. Radiat. Res., April 2021; 19(2): 299-308

DOI: $10.29252 /$ ijrr.19.2.299

\begin{abstract}
Background: Single photon emission computed tomography (SPECT)-alone imaging using the Tc-99m radiopharmaceutical labeled with methylene diphosphonate or similar analogs is usually employed to diagnose metastatic bone and is typically followed by complementary magnetic resonance (MR) imaging for support in clinical decision-making. In this study, two attenuation map generation approaches from MR and SPECT non-attenuation corrected (SPECT-nonAC) images were evaluated in the context of quantitative SPECT imaging. Materials and Methods: The 2class-MR attenuation map was generated via segmenting an MR image into air and soft tissue. Likewise, SPECT-nonAC was segmented into background air and soft tissue to generate a 2class-SPECT attenuation map. The reference attenuation map was generated through manual bone segmentation from an MR image to develop a 3class-bone attenuation map. Standard uptake value (SUV) bias was calculated using the different attenuation maps on 50 vertebrae from normal patients and 16 vertebrae from metastatic patients. Results: The 2class-MR approach resulted in $-16 \%$ and $-8 \%$ SUV bias in normal and metastatic groups, respectively, while 2 class-SPECT led to $33 \%$ and $26 \%$ SUV underestimation for the normal and metastatic patient groups, respectively. Conclusion: The 2class-SPECT approach led to a significant underestimation of SUV due to the uncertainty of body contour delineation. However, the 2class-MR approach resulted in less than $-9 \%$ SUV bias in metastatic patients, demonstrating its potential to support quantitative SPECT imaging.
\end{abstract}

Keywords: SPECT, attenuation correction, quantitative imaging, MR.

\section{INTRODUCTION}

Bone metastasis is a common type of metastasis that usually located on the spine (1-4). Due to varying bone reactions to metastases, different techniques have been developed to accurately detect and monitor metastasis $(3,5,6)$. Single-photon emission computed tomography (SPECT) is an effective diagnostic method related to bone metastases, owing to its capability to determine the precise location of bone lesions and to determine the status of metastases $(1,7)$. Nevertheless, the interpretation of SPECT images, particularly for bone metastases, is usually performed via visual inspections by nuclear medicine specialists, wherein the quantitative aspect of the SPECT imaging plays a minor role ${ }^{(8-10)}$. This has been 
considered one of SPECT's limitations when compared to positron emission tomography (PET) imaging (1). This limitation is mostly due to the fact that quantitative SPECT imaging requires correction for some degrading physical factors such as photon attenuation and scattering $(11,12)$.

The standard uptake value (SUV) indicator has been widely used to quantify normal tissue and tumor functional activities in quantitative PET imaging. However, recently, this semi-quantitative metric has also been used in SPECT imaging, especially for bone scan studies such as knee osteoarthritis (13), normal vertebrae (14) and differentiating bone metastases caused by degenerative changes in patients with prostate cancer (15).

Correction for attenuated photons, which may cause image artifacts and skew the visual interpretation of SPECT images, plays a key role in quantitative SPECT imaging $(16,17)$. In hybrid SPECT-CT scanners, an accurate attenuation map is readily provided by anatomical CT imaging. However, due to the additional dose of ionizing radiation and the poor soft-tissue contrast of CT imaging, alternative anatomical magnetic resonance (MR) imaging has been utilized in hybrid scanners (18). Though MR imaging does not impose additional radiation and enables high-contrast imaging of soft-tissues, MR signals do not directly provide electron density information. Thus, it is still very challenging to generate an attenuation map from MR when using SPECT-MR or PET-MR hybrid systems $(18,19)$. Previously proposed techniques for estimating attenuation maps from MR images can be categorized into three general types (20): a) tissue segmentation (which involves the bulk segmentation of MRI into a number of tissue classes) (21), b) the template-based method (which employs a number of atlas images to predict the attenuation map for the target MRI through image registration) (22-24), and c) machine learning (which utilizes artificial intelligence to train an algorithm to directly produce an attenuation map from MR images) $(25,26)$.

99mTc methylene diphosphonate (MDP) SPECT imaging is one of the standard methods for the initial assessment of bone tumors; however, its anatomical detail, sensitivity, and specificity are limited (27). Complementary MR imaging allows for visualization of the bone marrow and abnormalities with high anatomic resolution (27). In our medical imaging center, (Yazd, Iran), SPECT-alone imaging, using a dual-head system (SPECT-dual head Philips ADAC, forte, Netherlands), is routinely used to perform 99mTc MDP scan for assessment of bone metastasis. Very often, patients are referred to a T2-weighted MR imaging in the lumbar vertebrae region. Nevertheless, the evaluation of the bone metastasis is potentially hindered by non-quantitative SPECT data due to a lack of attenuation correction. To overcome this barrier, we set out to assess the accuracy of the two 2-tissue class attenuation maps in 99mTc MDP SPECT imaging in terms of SUV bias for patients with and without metastases. This is of particular interest for the molecular imaging centers, where SPECT-only scans are performed for diagnosis of the metastatic bone followed by a complementary MR imaging. This study investigates the benefits of employing the complementary MR images for the task of attenuation correction in order to perform quantitative SPECT imaging (quantitative parameters extracted from 99mTc SPECT images) for both normal patients and patients with bone metastasis. Although there are numerous studies in the literature concerning the MR-guided attenuation correction in PET imaging $(20,28,29)$, less attention has been paid to the quantitative SPECT imaging, particularly the impact of the MR-guided attenuation correction.

\section{MATERIALS AND METHODS}

\section{Patient population}

The clinical dataset of this study is derived from 16 patients (seven women and nine men, mean age: $57.2 \pm 16.3$, age range: $28-82$ ) who were referred to $99 \mathrm{mTc}$ MDP bone scintigraphy of the lumbar region. This study was conducted with the approval of the Ethics Committee of Shahid Sadoughi University of Medical Sciences under the Ethics code IR.SSU.MEDICINE.REC. 
1396.158, with a registration date of $1396 / 07 / 12$ or $04 / 10 / 17$.

The injected activity of $99 \mathrm{mTc}$ ranged from 740 to $1110 \mathrm{MBq}$ (mean797 $\pm 97 \mathrm{MBq}$ ), with a mean injected activity of $12.9 \pm 1.8 \mathrm{MBq} / \mathrm{kg}$. The planar scanning and SPECT images of each patient were obtained within $3.5 \pm 1$ hours after the intravenous injection (30). The patient data employed in this study were divided into normal (10 patients) and metastatic (6 patients) groups. Patients in the metastatic group had at least one metastatic lesion in one of their lumbar vertebrae.

\section{Clinical data acquisition}

The SPECT images were acquired with a dual-head SPECT system using low-energy high-resolution collimation, 32 projections over $360^{\circ}, 20$ seconds acquisition at each projection, an energy window of $\pm 10 \%$ for scattered photon removal and image matrix of $128 \times 128$ with 4.72 $\mathrm{mm}$ pixel size.

The T2-weighted images of the patients were acquired using a MAGNETOM MRI scanner (Siemens MAGNETOM Avanto 1.5T, Germany), with a repetition time (TR) of $3080 \mathrm{~ms}$, echo time (TE) of $103 \mathrm{~ms}$, a flip angle (FA) of 150 degrees and a matrix size of $320 \times 290$.

\section{Attenuation map generation}

In this study, three different attenuation maps were generated; two using the T2 MR Image and one from SPECT non-attenuation corrected (SPECT-nonAC). Since the MR and SPECT scanning was performed sequentially, in the first step, the MR images were non-rigidly aligned to the SPECT images. The image alignment was carried out using Medical Image Processing, Analysis and Visualization (MIPAV) software (version 8.0.1-USA) based on thin-plate spline algorithm (31) for the non-rigid registration. Since SPECT images do not contain sufficient anatomical information, a number of anatomical landmarks were manually defined on both SPECT and MR images to assist the registration process.

3class-bone attenuation map: an MR-derived attenuation map with a separate class for bony tissues was created, wherein the MR images were segmented into three tissue classes; air, soft-tissue, and bone. The air class involves the back-ground air as well as the stomach air pockets. To take bone into account, the ribs and vertebral bodies were manually segmented and classified as bone tissue class. The rest of the body inside the body contour was considered as soft-tissue (figure 1.c). The predefined attenuation coefficients of $0,0.15$, and $0.26 \mathrm{~cm}^{-1}$ were assigned to air, soft-tissue, and bone, respectively.

2class attenuation map from MR (2class-MR): Manual bone delineation from MR images is a tedious and prohibitively time-consuming task (32, 33). Alternatively, MR images can be segmented into a 2class attenuation map representing air and soft-tissue. To this end, the body contour was segmented from the MR image and the voxels inside the body contour were assigned to the soft-tissue. Similarly, the voxels outside the body contour were considered as air (figure 1.d).

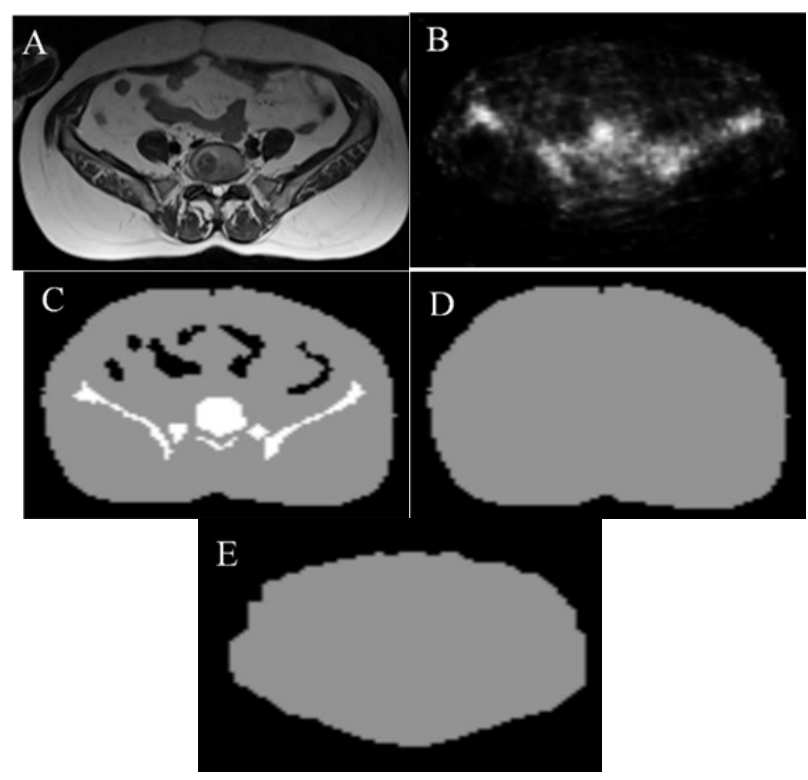

Figure 1. Representative axial slices of a) MR image, b) SPECT -nonAC, c) 3class-bone, d) 2class-MR, and e) 2class-SPECT of a single patient.

2class attenuation map from SPECT (2classSPECT): A 2-class attenuation map could also be generated from the SPECT-nonAC image when MR image does not exist or when there is a 
massive truncation in the MR image. Similar to the 2class-MR approach, the body contour in each slice was delineated from SPECT image followed by the assignment of air and soft-tissue attenuation value to the voxels outside and inside the body contour, respectively (figure 1.e).

The attenuation coefficients of the tissues were obtained as follows: first, the elemental composition of each tissue together with its mass percentage and tissue density were obtained from the International Commission on Radiation Units and Measurements (ICRU) report 44 (34). Then, based on their elements, the mass percentage, and the energy range of the employed radionuclide $(0.14 \mathrm{MeV})$, a value was extracted from XCOM photon cross-section library (35). This value was then multiplied with the mass density of each tissue to calculate the linear attenuation coefficient of the tissue.

\section{SPECT reconstruction}

Reconstruction of SPECT images were performed using the Ordered Subset Expectation Maximum (OSEM); the one step late algorithm was implemented in the open-source STIR libraries (Software for Tomographic Image Reconstruction, Version 3) (36). In this study, the reconstruction was repeated four times using 3class-bone (as reference), 2class-MR, 2class-SPECT and without attenuation correction (SPECT-nonAC). The reconstructions were carried out using 20 iterations and 8 subsets.

\section{Evaluation strategy}

Fifty normal vertebrae from the normal patient group were manually segmented to estimate the SUV within each vertebra. In six metastatic patients, only 13 out of 30 lumbar vertebrae with the increased uptake were observed and were classified as metastatic. The SUV and activity concentration (ACC) in $\mathrm{kBq} / \mathrm{mL}$ unit were calculated for volumes of interest (VOIs) drawn on the metastatic and normal vertebrae, separately.

Elliptical VOIs with a fixed volume of $14.3 \mathrm{~mL}$ were manually defined on the vertebrae of normal and metastatic patients, in a way to include a large part of the spongy bone tissue while avoiding adjacent vertebral disc. Moreover, a VOI of $0.946 \mathrm{~mL}$ was drawn on a single slice of each vertebral body, which contained the highest mean count for the SUV peak calculation.

In order to extract quantitative information from the SPECT images (SUV estimation), it is necessary to calculate the calibration factor (conversion factor) to estimate absolute activity concentration from raw counts data. The count rate correction factors were calculated by acquiring the image of a point source ${ }^{99 \mathrm{~m}} \mathrm{Tc}$ with the fixed activity of $37 \mathrm{MBq}$ in the air (37). Then, the SUV $V_{\mathrm{BW}}$ (body weight) and SUV $\mathrm{LBM}_{\mathrm{LB}}$ (lean body mass) values were calculated based on body weight for $\mathrm{SUV}_{\text {mean, }} \mathrm{SUV}_{\text {peak, }} \mathrm{SUV}_{\text {max }}$ and lean body mass for $\mathrm{SUV}_{\text {mean, }}$ respectively (38). The following equations were used for the calculation of $S_{U V} V_{B W}$ (Eq. 1) and $S U V_{L B M}$ (Eq. 2).

$$
\begin{aligned}
& \text { SUV }_{\text {BW }}=\frac{\text { ACC } \times \text { Weight }}{\text { Injected Activity }} \\
& \mathrm{SUV}_{\text {LBM }}=\frac{\text { ACC } \times \text { LBM }}{\text { Injected Activity }}
\end{aligned}
$$

For males: $L B M=1.10 \times$ weight $(\mathrm{kg})-128 \times$ weight $(\mathrm{kg})^{2} /$ height $\left.(\mathrm{cm})^{2}\right)^{(38)}$

For females: $L B M=1.07 \times$ weight $(\mathrm{kg})-148 \times$ weight $(\mathrm{kg})^{2} /$ height $\left.(\mathrm{cm})^{2}\right)^{(38)}$

The 3class-bone attenuation map was considered as a reference, against which the 2class-MR and 2class-SPECT methods were evaluated. Eq. 3 was used to calculate the relative errors for SUV and ACC values.

Relative error $(\%)=$ $\frac{\text { ACC or SUV(2class_MRI or 2class_SPECT)-ACCorSUV(3class_bone) }}{\text { ACC or SUV(3class_bone) }} \times 100$

The correlation between SUVs and ACC values estimated on the SPECT images corrected using 2class-MRI and 2class-SPECT attenuation maps versus the reference 3class-bone attenuation map were examined for normal and metastatic patients.

Int. J. Radiat. Res., Vol. 19 No. 2, April 2021 


\section{Statistical analysis}

The estimated SUV and ACC associated with each vertebra was considered as a single data point to create the scatter plot, it was also used for linear regression analysis. The distribution of these values was analyzed using Shapiro-Wilk and Kolmogorov-Smirnov normalization tests. The independent samples T-test and Mann-Whitney U-test were also used to assess the difference between ACC values and SUV $_{\text {mean }}$ of the normal and metastatic groups employing SPSS ver. 16.0 (IBM Corp.) software. The p-values less than 0.05 were considered as statistically significant.

\section{RESULTS}

In this study, the 3class-bone AC map was considered as a reference based on the quantitative accuracy of the 2class-MRI and 2class-SPECT AC maps, which were investigated for SPECT attenuation correction. To this end, the accuracy of the SUVs obtained by applying these two AC maps was compared against the 3class-bone for normal patients and patients with bone metastases in MDP SPECT imaging.

Figure 1 shows representative slices of the 2class-MRI, 2class-SPECT, and 3class-bone attenuation maps, as well as the original MR and SPECT-nonAC images of a patient with bone metastasis. Figure 2 presents the planar scintigraphy of the same patient depicted in figure 1 , as well as the corresponding axial views of the SPECT images after they were corrected for attenuation using 2class-MRI, 2class-SPECT, and 3class-bone attenuation maps.

Table 1 presents a statistical summary of the quantitative evaluation of SPECT images corrected for photon attenuation using the three different attenuation maps for normal and metastatic patient groups. For the normal group, the $S_{U V} V_{\text {mean }}$ measured on the vertebra bodies in the SPECT images decreased moderately from 3.64 (when using 3class-bone) to 3.05 (when using 2class-MR). The same measurement on SPECT images corrected by 2class-SPECT exhibited a significant under-estimation of activity concentration, leading to a SUV $V_{\text {mean }}$ of
2.42. A similar trend was observed in metastatic

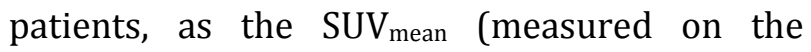
metastatic vertebrae) decreased from 10.42 to 9.41 when using the 3class-bone and 2class-MR AC maps, respectively. However, the 2class-SPECT approach produced a significant SUV under-estimation, resulting in a $S_{U} V_{\text {mean }}$ of 7.52. This difference is more noticeable when considering the $\mathrm{SUV}_{\max }$ of the metastatic patients, as an SUV $V_{\max }$ of 15.25 was observed in 3class-bone SPECT images (versus 13.63 and 10.65 obtained from the 2class-MR and 2class-SPECT approaches, respectively).

Figure 3 illustrates the mean and standard deviation of SUV and ACC values estimated on the SPECT images for 50 vertebrae (including ten samples for each of 1st lumbar vertebra (L1), 2nd lumbar vertebra (L2), 3rd lumbar vertebra (L3), 4th lumbar vertebra (L4), and 5th lumbar vertebra (L5)) in the normal patient group. Similarly, for metastatic patients, the measurements were performed on 13 vertebrae (including two L1 samples, two L2 samples, four L3 samples, one L4 sample, and four L5 samples).

Figure 4 displays the results of the linear regression analysis and the correlation between the SUV and ACC measured on the SPECT images corrected for attenuation by 2class-MR and 2class-SPECT AC maps and compares them to the reference 3class-bone approach in the normal patient group. Similarly, Figure 5 presents the results of the same analysis for the metastatic patient group. The regression plots exhibited a good correlation $\left(\mathrm{R}^{2}=0.93\right.$ for $\mathrm{SUV}_{\text {mean, }} 0.87$ for ACC) between the 3class-bone and 2class-MR AC techniques. However, a weak correlation $\left(\mathrm{R}^{2}=0.65\right.$ for $\mathrm{SUV}_{\text {mean }}, 0.54$ for ACC) was observed between 2class-SPECT and 3class-bone AC techniques for the normal group. On the other hand, the regression plots for the metastatic group demonstrated a good correlation $\left(\mathrm{R}^{2}=0.93\right.$ for $\mathrm{SUV}_{\text {mean, }} 0.95$ for ACC) between the 3class-bone and 2class-MR AC techniques. However, in contrast to the normal patient group, the 2class-SPECT approach resulted in a relatively good correlation $\left(\mathrm{R}^{2}\right.$ $=0.83$ for $\mathrm{SUV}_{\text {mean }}, 0.90$ for ACC) with the 3class-bone approach. 
The 2class-MR and 2class-SPECT AC maps led to $\mathrm{SUV}_{\text {mean }}$ underestimations of $16.21 \%$ and $33.52 \%$, respectively. The 2class-SPECT and 2class-MR AC maps led to $\mathrm{SUV}_{\text {mean }}$
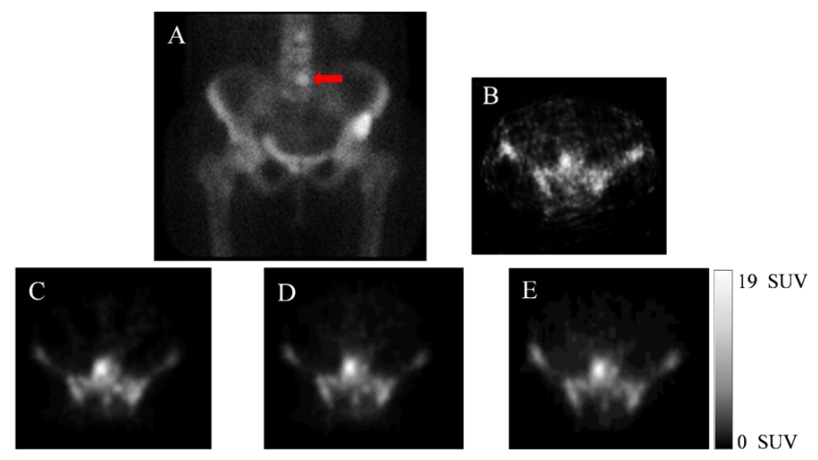

Figure 2. a) Planar image of a patient with bone metastasis (the arrow indicates a metastatic lesion). Axial views of the fifth lumbar vertebral in b) SPECT-nonAC and the SPECT images attenuation corrected using; c) 3class-bone, d) 2class-MR and e) 2class-SPECT attenuation maps.
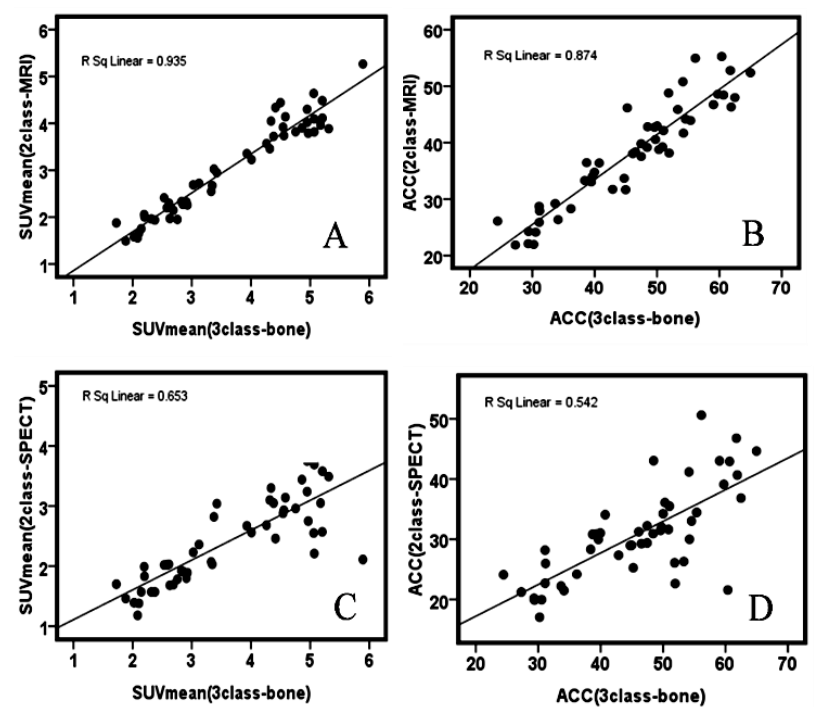

Figure 4. The linear regression plots of correlation between the SPECT images attenuation corrected using the 2class-MR and 2 class-SPECT versus the reference 3 class-bone attenuation maps. A) 2class-MR vs. 3class-bone (SUVmean). B) 2class-SPECT vs. 3class-bone (ACC). C) 2class-SPECT vs. 3class-bone (SUVmean). D) 2class-SPECT vs. 3class-bone (ACC). Fifty data points represent SUVmean and ACC of 5 lumbar vertebrae in normal patient group. underestimations of $26.56 \%$ and $8.11 \%$, respectively. The underestimation of SUV was reduced in the metastatic group when compared to the normal group.
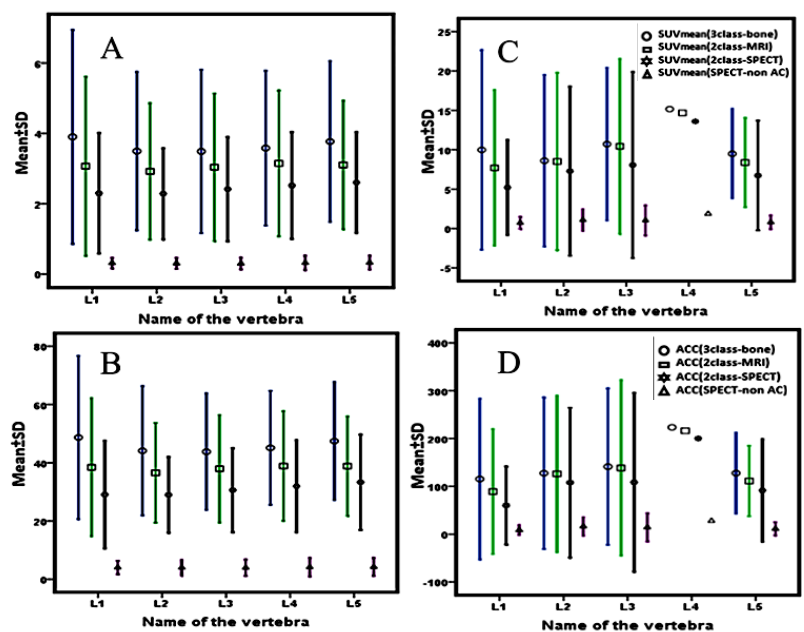

Figure 3. A) SUVmean, B) ACC values for the 5 vertebrae (L1 to L5) calculated across 10 normal patients, C) SUVmean and D) ACC values for the 5 vertebrae (L1 to L5) calculated across 6 metastatic patients.
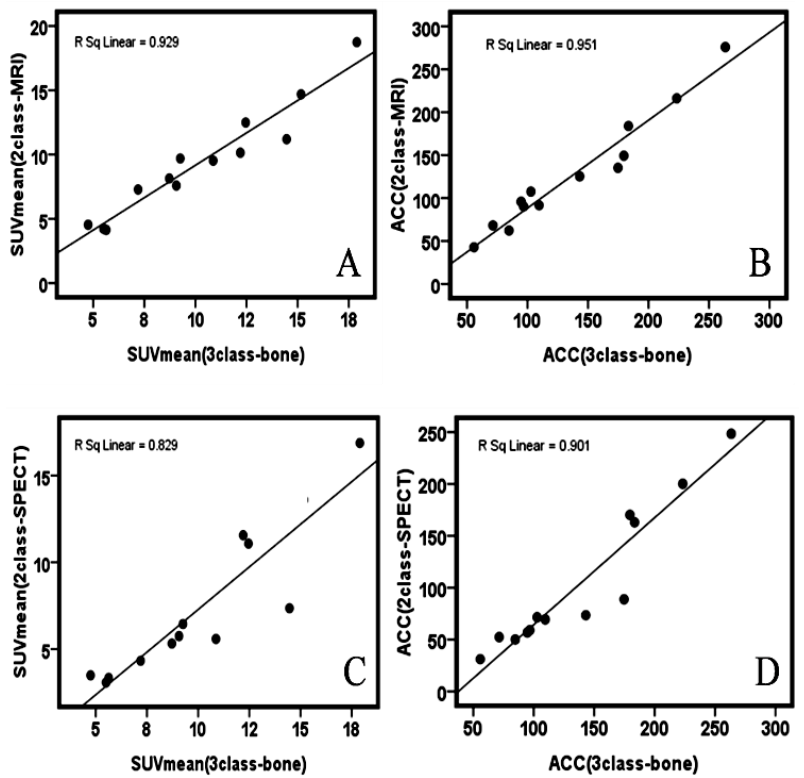

Figure 5. The linear regression plots of correlation between the SPECT images attenuation corrected using the 2class-MR and 2 class-SPECT versus the reference 3 class-bone attenuation maps. A) 2class-MR vs. 3class-bone (SUVmean). B) 2class-SPECT vs. 3 class-bone (ACC). C) 2 class-SPECT vs.

3 class-bone (SUVmean). D) 2class-SPECT vs. 3class-bone (ACC). Thirteen data points represent SUVmean and ACC of 5 lumbar vertebrae in metastatic patient group. 
Table 1. Statistical summary of SUV and activity measurement for 50 vertebral bodies (normal patient group) and 13 vertebral bodies (metastatic patient group) in the SPECT images attenuation corrected using different methods.

\begin{tabular}{|c|c|c|c|c|c|c|c|c|c|}
\hline \multirow{2}{*}{\multicolumn{2}{|c|}{ Variable }} & \multicolumn{2}{|c|}{$\begin{array}{l}\text { 3class-bone } \\
\text { (Mean } \pm S D)\end{array}$} & \multicolumn{2}{|c|}{$\begin{array}{l}\text { 2class-MRI } \\
\text { (Mean } \pm S D)\end{array}$} & \multicolumn{2}{|c|}{$\begin{array}{c}\text { 2class-SPECT } \\
\text { (Mean } \pm S D)\end{array}$} & \multicolumn{2}{|c|}{$\begin{array}{c}\text { SPECT-non AC } \\
\text { (Mean } \pm \text { SD) }\end{array}$} \\
\hline & & normal & metastatic & normal & metastatic & normal & metastatic & normal & metastatic \\
\hline \multicolumn{2}{|c|}{$S U V_{\text {peak }}$} & $4.56 \pm 1.59$ & $14.05 \pm 5.31$ & $3.80 \pm 1.36$ & $12.45 \pm 5.29$ & $3.06 \pm 0.93$ & $9.79 \pm 5.40$ & $0.40 \pm 0.14$ & $1.36 \pm 0.84$ \\
\hline \multicolumn{2}{|c|}{$S U V_{\max }$} & $5.03 \pm 1.80$ & $15.25 \pm 5.75$ & $4.18 \pm 1.56$ & $13.63 \pm 5.71$ & $3.34 \pm 1.05$ & $10.65 \pm 5.68$ & $0.41 \pm 0.15$ & $1.39 \pm 0.86$ \\
\hline \multicolumn{2}{|c|}{$S U V_{\text {mean }}$} & $3.64 \pm 1.18$ & $10.24 \pm 4.05$ & $3.05 \pm 1.01$ & $9.41 \pm 4.24$ & $2.42 \pm 0.73$ & $7.52 \pm 4.38$ & $0.31 \pm 0.08$ & $0.98 \pm 0.64$ \\
\hline \multicolumn{2}{|c|}{$S U V_{\text {mean LMB }}$} & $2.62 \pm 0.73$ & $8.05 \pm 3.48$ & $2.19 \pm 0.63$ & $7.40 \pm 3.64$ & $1.76 \pm 0.53$ & $5.98 \pm 3.79$ & $0.23 \pm 0.08$ & $0.79 \pm 0.55$ \\
\hline \multicolumn{2}{|c|}{$\mathrm{ACC}(\mathrm{kBq} / \mathrm{ml})$} & $45.82 \pm 10.82$ & $137.15 \pm 63.13$ & $38.14 \pm 9.24$ & $126.47 \pm 66.24$ & $30.79 \pm 7.73$ & $102.65 \pm 68.63$ & $4.09 \pm 1.36$ & $13.55 \pm 10.03$ \\
\hline \multicolumn{2}{|c|}{ Age(year) } & \multicolumn{2}{|c|}{ Weight(kg) } & \multicolumn{2}{|c|}{ Height(cm) } & \multicolumn{2}{|c|}{$\begin{array}{c}\text { Administered activity } \\
\text { (MBq) }\end{array}$} & \multicolumn{2}{|c|}{$\begin{array}{l}\text { Activity per } 1 \mathrm{~kg} \text { BW } \\
(\mathrm{MBq} / \mathrm{kg})\end{array}$} \\
\hline normal & metastatic & normal & metastatic & normal & metastatic & normal & metastatic & normal & metastatic \\
\hline $55 \pm 15.34$ & $64.15 \pm 17.9$ & $64.10 \pm 15.68$ & $58.77 \pm 10.89$ & $161.70 \pm 8.1$ & $159.69 \pm 8.19$ & $814 \pm 119.58$ & $762.78 \pm 32.18$ & $13.05 \pm 1.92$ & $13.28 \pm 1.76$ \\
\hline
\end{tabular}

\section{DISCUSSION}

The quantification of the SPECT images (either from SPECT-alone or SPECT-MR imaging) is hindered by physical deterioration factors such as photon attenuation and scattering. In recent years, some researchers have studied the quantification of SPECT images by measuring the SUV in SPECT-CT bone scans. Cachovan et al. (12) determined the ACC and SUV of the concentration of $99 \mathrm{~m} \mathrm{Tc}$-DPD (diphosphono -propanedicarboxylic acid) obtained from SPECT-CT images of healthy lumbar vertebrae. In 2016, Kaneta et al. (14) performed a quantitative analysis based on the SUV of the uptake of $99 \mathrm{mTc}$-MDP in normal vertebrae using a SPECT-CT scanner. Also, in 2019, Mohd Rohani et al. (39) evaluated the SUV of a 99mTc -MDP radionuclide tracer in the normal vertebrae of breast cancer patients using a SPECT-CT scanner.

In the present study, the quantitative accuracy of the SPECT imaging using two 2-tissue class attenuation maps obtained from MR (for SPECT/MR imaging) and SPECT-nonAC (for SPECT-alone imaging) images was evaluated against a 3-tissue class attenuation map involving bone tissue in terms of SUV and ACC. The larger bias observed in 2class-SPECT $\mathrm{AC}$ is due to the underestimated body contour (smaller than the actual size) obtained from the SPECT-nonAC images. The underestimation of the body contour is visible in figure 1 when Int. J. Radiat. Res., Vol. 19 No. 2, April 2021 compared to the MR image and 2class-MR attenuation map. The detection of body borders is very challenging in SPECT images due to the very weak signals and low activity concentration, particularly when $99 \mathrm{~m}$ Tc labeled with MDP is administered. The fat tissue in MDP SPECT has very low SNR, as the radiotracer is mostly absorbed by the bony tissue.

2class-MR and 2class-SPECT AC maps resulted in $16.21 \%$ and $33.52 \%$ underestimations of $S U V_{\text {mean, }}$, respectively. These underestimations primarily stem from a lack of bony tissue in these 2-class attenuation maps, particularly for VOIs drawn within bony tissues. A similar negative SUV bias was observed in PET/MR imaging when attenuation maps with no separate class for bone were used (21). Kim et al. (40) showed that for malignant lesions in the spine, the SUVs measured from PET images corrected for attenuation without considering bony tissue were underestimated by $16.4 \pm$ $8.5 \%$. Despite the noticeable negative bias of the 2class-MR approach, the mean $S_{\text {mean }}$ difference between 2class-MR and 3class-bone $\mathrm{AC}$ is less than 0.6, while the 2class-SPECT produced a mean $S V_{\text {mean }}$ difference of more than 1.2.

The absolute SUVs measured from SPECT images corrected using the 3class-bone approach for normal patients are in agreement with the values estimated from the SPECT-CT images in the literature (e.g., an $\mathrm{SUV}_{\text {mean }}$ of $3.92 \pm 0.27$ was reported by Mohd Rohani et al., 
an $\mathrm{SUV}_{\text {mean }}$ of $4.4 \pm 0.5$ was estimated by Kaneta et al. (14), and an ACC of $48.15 \pm 13.66$ was produced by Cachovan et al. (12) for a number of VOIs defined in the spongy region of the vertebrae and the entire vertebra volume). Despite the agreement between the SUVs estimated from CT and 3class-bone AC approaches, a major limitation of the current study is the lack of ground-truth CT attenuation maps.

The linear attenuation coefficients used in this study are considered narrow beam attenuation coefficients since the attenuation correction was applied without any scatter correction. However, in order to minimize the impact of scattering, an energy window of $\pm 10 \%$ was applied during the SPECT acquisition. Using the narrow beam attenuation coefficients would result in an overestimation of the activity concentration (41). However, this condition was the same for the entire set of reconstructed SPECT images using different attenuation maps.

Attenuation maps generated from MR images and acquired non-concurrently with SPECT acquisition are subject to imperfect image alignment. Automatic inter-modality image registration is challenged by a lack of sufficient anatomical landmarks. Moreover, discrepancies in patient positioning and respiration phases between the two modalities add to the complexity of this issue. Attenuation maps generated directly from SPECT images can eliminate the need for inter-modality image registration. However, a quantitative analysis of this study demonstrated that under-estimations of the body contour obtained from SPECT-nonAC (when ${ }^{99 \mathrm{mTc}} \mathrm{MDP}$ is administrated) can lead to significant relative bias (up to 33\%). In cases when an MR image does not exist, or when there is a gross truncation in MR images, special attention should be paid to body contouring from the SPECT images to determine the entire body volume.

\section{CONCLUSION}

The MR images obtained sequentially after Tc -99m MDP-SPECT imaging could be used to generate an attenuation map for the task of quantitative SPECT imaging. The MR-derived AC map resulted in less than $-9 \%$ SUV bias in quantitative lesion analysis performed on the SPECT images. Therefore, MR-derived AC maps could support quantitative SPECT imaging in the absence of the gold-standard of CT-based attenuation correction.

\section{Compliance with ethical standards}

Funding sources: This research did not receive any specific grant from funding agencies in the public, commercial, or not-for-profit sectors.

Ethical approval: The analysis of patients' data was performed with the permission of the Ethics Committee of our University.

Informed consent: Informed consent was obtained from all individual participants included in the study in order to investigate their images for this project.

\section{Conflicts of interest: Declared none.}

\section{REFERENCES}

1. O'Sullivan GJ, Carty FL, Cronin CG (2015) Imaging of bone metastasis: An update. World J Radiol, 7(8): 202-11.

2. Guillevin R, Vallee JN, Lafitte F, Menuel C, Duverneuil NM, Chiras J ( 2007) Spine metastasis imaging: review of the literature. J Neuroradiol, 34(5): 311-21.

3. Beheshti M, Langsteger W, Fogelman I ( 2009) Prostate cancer: role of SPECT and PET in imaging bone metastases. Semin Nucl Med, 39(6): 396-407.

4. An C, Lee YH, Kim S, Cho HW, Suh JS, Song HT ( 2013;) Characteristic MRI findings of spinal metastases from various primary cancers: retrospective study of pathologicallyconfirmed cases. Journal of the Korean Society of Magnetic Resonance in Medicine, 17(1): 8-18.

5. Ulmert D, Solnes L, Thorek D (2015) Contemporary approaches for imaging skeletal metastasis. Bone Res, 3: 15024.

6. Shah LM and Salzman KL (2011) Imaging of spinal metastatic disease. Int J Surg Oncol, 2011: 769753.

7. Heindel W, Gubitz R, Vieth V, Weckesser M, Schober O, Schafers M (2014) The diagnostic imaging of bone metastases. Dtsch Arztebl Int, 111(44): 741-7.

Int. J. Radiat. Res., Vol. 19 No. 2, April 2021 
8. Yahara J, Noguchi M, Noda S (2003) Quantitative evaluation of bone metastases in patients with advanced prostate cancer during systemic treatment. BJU Int, 92(4): 379-84.

9. Kaboteh R. Quantitative analysis of bone scans in prostate cancer patients [dissertation]. [Sweden]: University of Gothenburg. Sahlgrenska Academy; 2013.62p.

10. Beck M, Sanders JC, Ritt P, Reinfelder J, Kuwert T (2016) Longitudinal analysis of bone metabolism using SPECT/CT and 99mTc-diphosphono-propanedicarboxylic acid: comparison of visual and quantitative analysis. EJNMMI Research, 6(1): 60.

11. Zeintl J, Vija AH, Yahil A, Hornegger J, Kuwert T (2010) Quantitative accuracy of clinical $99 \mathrm{mTC}$ SPECT/CT using ordered-subset expectation maximization with 3dimensional resolution recovery, attenuation, and scatter correction. J Nucl Med, 51(6): 921.

12. Cachovan M, Vija AH, Hornegger J, Kuwert T (2013) Quantification of $99 \mathrm{~m}$ Tc-DPD concentration in the lumbar spine with SPECT/CT. EJNMMI research, 3(1): 45.

13. Kim J, Lee H-H, Kang $Y$, Kim T, Lee S, So $Y$, et al. (2017) Maximum standardised uptake value of quantitative bone SPECT/CT in patients with medial compartment osteoarthritis of the knee. Clin Radiol, 72(7): 580-9.

14. Kaneta T, Ogawa M, Daisaki H, Nawata S, Yoshida K, Inoue T (2016) SUV measurement of normal vertebrae using SPECT/CT with Tc-99m methylene diphosphonate. Am J Nucl Med Mol Imaging, 6(5): 262-268

15. Kuji I, Yamane T, Seto A, Yasumizu Y, Shirotake S, Oyama M (2017) Skeletal standardized uptake values obtained by quantitative SPECT/CT as an osteoblastic biomarker for the discrimination of active bone metastasis in prostate cancer. European Journal of Hybrid Imaging, 1(1): 2.

16. Buvat I, Frey E, Green A, Ljungberg M (2014) Quantitative nuclear medicine imaging: concepts, requirements and methods. Human Health Reports, 9: 59

17. Marshall HR, Stodilka RZ, Theberge J, Sabondjian E, Legros A, Deans L, et al. (2011) A comparison of MR-based attenuation correction in PET versus SPECT. Phys Med Biol, 56 (14): 4613-29.

18. Bouziotis P and Fiorini C (2014) SPECT/MRI: dreams or reality? Clinical and Translational Imaging, 2(6): 571-3.

19. Arabi H, Dowling JA, Burgos N, Han X, Greer PB, Koutsouvelis $N$, et al. (2018) Comparative study of algorithms for synthetic CT generation from MRI: Consequences for MRIguided radiation planning in the pelvic region. Med Phys, 45(11): 5218-33.

20. Mehranian A, Arabi H, Zaidi H ( 2016) Magnetic resonance imaging-guided attenuation correction in PET/MRI: Challenges, solutions, and opportunities. Med Phys, 43(3): 1130-55.

21. Arabi H, Rager O, Alem A, Varoquaux A, Becker M, Zaidi H (2015) Clinical assessment of MR-guided 3-class and 4class attenuation correction in PET/MR. Molecular Imaging and Biology, 17(2): 264-76.

22. Arabi H, Zaidi H (2016) One registration multi-atlas-based pseudo-CT generation for attenuation correction in PET/ MRI. Eur J Nucl Med Mol Imaging. 43(11): 2021-35.
23. Arabi $H$ and Zaidi H (2016) Magnetic resonance imagingguided attenuation correction in whole-body PET/MRI using a sorted atlas approach. Medical Image Analysis, 31: 1-15.

24. Arabi $H$, Koutsouvelis N, Rouzaud M, Miralbell R, Zaidi $H$ (2016) Atlas-guided generation of pseudo-CT images for MRI-only and hybrid PET-MRI-guided radiotherapy treatment planning. Physics in medicine and biology, 61(17): 6531.

25. Arabi H, Zeng G, Zheng G, Zaidi H (2019) Novel adversarial semantic structure deep learning for MRI-guided attenuation correction in brain PET/MRI. Eur J Nucl Med Mol Imaging, 46(13):2746-2759.

26. Han X (2017) MR-based synthetic CT generation using a deep convolutional neural network method. Medical Physics, 44(4): 1408-19.

27. Steinborn $M M$, Heuck $A F$, Tiling $R$, Bruegel $M$, Gauger $L$, Reiser MF( 1999) Whole-body bone marrow MRI in patients with metastatic disease to the skeletal system. J Comput Assist Tomogr, 23(1): 123-9.

28. Mehranian A, Arabi H, and Zaidi H (2016) Quantitative analysis of MRI-guided attenuation correction techniques in time-of-flight brain PET/MRI. Neuroimage, 130: 123133.

29. Bortolin K, Arabi H, and Zaidi H (2019) Deep learningguided attenuation and scatter correction without using anatomical images in brain PET/MRI. IEEE Nuclear Science Symposium and Medical Imaging Conference (NSS/MIC), II1-3. United Kingdom.

30. Van den Wyngaert T, Strobel K, Kampen WU, Kuwert T, van der Bruggen W, Mohan HK, et al. (2016) The EANM practice guidelines for bone scintigraphy. Eur J Nucl Med Mol Imaging, 43(9): 1723-38.

31. Qiu Z, Tang H, Tian D (2009) Non-rigid medical image registration based on the thin-plate spline algorithm. 2009 WRI World Congress on Computer Science and Information Engineering: IEEE.

32. Arabi $\mathrm{H}$ and Zaidi $\mathrm{H}$ (2016) Whole-body bone segmentation from MRI for PET/MRI attenuation correction using shape-based averaging. Med Phys, 43(11): 5848.

33. Arabi $\mathrm{H}$ and Zaidi $\mathrm{H}$ (2017) Comparison of atlas-based techniques for whole-body bone segmentation. Medical image analysis, 36: 98-112.

34. White DR, Wilson IJ, Booz J, Spokas JJ, Griffith RV (2016) Report 44. J Int Comm Radiat Units Measure, os23(1): NP$N P$.

35. Berger MJ, Hubbell JH (1987) XCOM: Photon Cross Sections Database, Web Version1.2, available at http:// physics.nist.gov/xcom., National Institute of Standards and Technology, Gaithersburg, MD 20899, USA.

36. Fuster BM, Falcon C, Tsoumpas C, Livieratos L, Aguiar $P$, Cot $A$, et al. (2013) Integration of advanced 3D SPECT modeling into the open-source STIR framework. Med Phys, 40(9): 092502.

37. Willowson K, Bailey DL, Baldock C (2008) Quantitative SPECT reconstruction using CT-derived corrections. Phys Med Biol, 53(12): 3099-112. 


\section{Mostafapour et al. / Attenuation map for quantitative SPECT imaging}

38. Hallynck T, Soep H, Thomis J, Boelaert J, Daneels R, Dettli L (1981) Should clearance be normalised to body surface or to lean body mass? British Journal of Clinical Pharmacology, 11(5): 523-6.

39. Rohani MFM, Yonan SNM, Tagiling N, Zainon WMNW, Udin Y, Nawi NM (2020) Standardized uptake value from semiquantitative bone single-photon emission computed tomography/computed tomography in normal thoracic and lumbar vertebrae of breast cancer patients. Asian Spine Journal, published online ahead of print(14(5): 629638).
40. Kim JH, Lee JS, Song IC, Lee DS (2012) Comparison of segmentation-based attenuation correction methods for PET/ MRI: evaluation of bone and liver standardized uptake value with oncologic PET/CT data. J Nucl Med, 53(12): 1878-82.

41. Grimes J. Patent-specifc internal dose calculaton techniques for clinical use in targeted radionuclide therapy [dissertation]. [Canada]: University of Britsh Columbia; 2013.186p 